\title{
Mastofauna terrestre do Parque Estadual da Serra do Tabuleiro, Estado de Santa Catarina, sul do Brasil
}

\author{
Jorge José Cherem ${ }^{1}$ Mauricio Eduardo Graipel ${ }^{2,3 *}$ Marcos Tortato $^{1}$

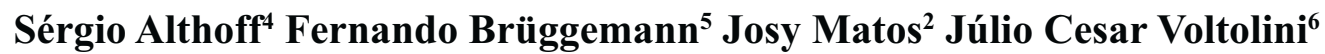 \\ Rodrigo Freitas $^{2}$ Rafael Illenseer ${ }^{2}$ Fernando Hoffmann ${ }^{1}$ Ivo Rohling Ghizoni-Jr. ${ }^{1}$ \\ Alexei Bevilacqua ${ }^{2}$ Rafael Reinicke $^{2}$ Carlos Henrique Salvador $^{1}$ Alexandre Filippini ${ }^{7}$ \\ Nina Furnari ${ }^{8}$ Karine Abati $^{2}$ Marcos Moraes ${ }^{2}$ Tiago Moreira ${ }^{2}$ Luiz Gustavo Rodrigues \\ Oliveira-Santos ${ }^{2}$ Vanessa Kuhnen ${ }^{2}$ Thiago Maccarini ${ }^{2}$ Fernando Goulart $^{2}$ Hugo Mozerle $^{2}$ Felipe $^{2}$ \\ Fantacini $^{2}$ Dayse Dias ${ }^{2}$ Rafael Penedo-Ferreira ${ }^{2}$ Bianca Pinto Vieira ${ }^{2}$ Paulo César Simões-Lopes ${ }^{3}$

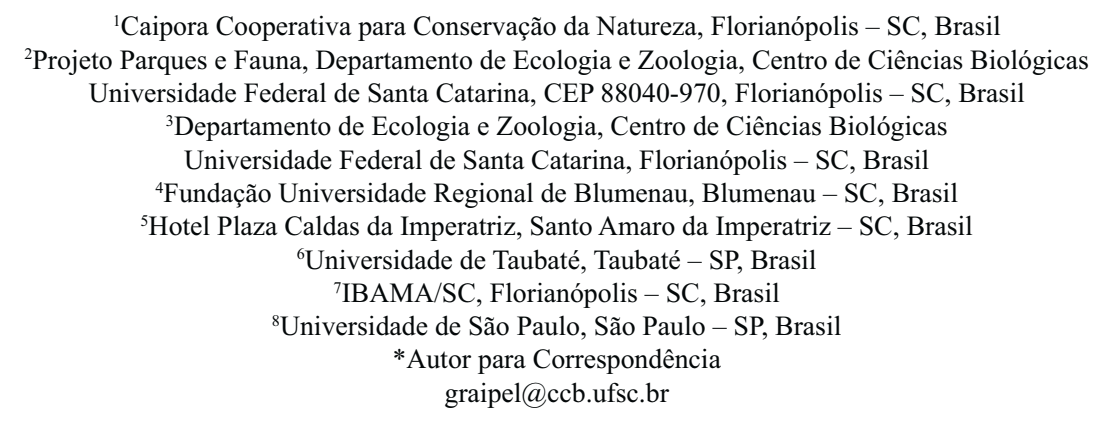

Submetido em 04/03/2011

Aceito para publicação em 21/06/2011

\section{Resumo}

O Parque Estadual da Serra do Tabuleiro, situado no centro-leste do Estado de Santa Catarina, sul do Brasil, possui 85.000 ha e abrange grande diversidade de ambientes (floresta ombrófila densa, incluindo manguezais e restingas, floresta ombrófila mista e estepe ombrófila). De 1991 a 2010 foram realizados 22 estudos sobre sua mastofauna, abrangendo os métodos de captura com armadilhas Young e de dupla entrada, armadilhas de interceptação e queda, caixas de nidificação, redes-neblina, armadilhas fotográficas, observação direta e vestígios. Foram registradas 75 espécies autóctones (cerca de 54\% das espécies de mamíferos terrestres confirmados para Santa Catarina) pertencentes a nove ordens e 25 famílias. Quinze espécies são ameaçadas em nível estadual, nacional ou global. Aspectos gerais da conservação dos mamíferos no Parque e a possibilidade de ocorrência de outras espécies são discutidos.

Palavras-chave: Conservação, Mamíferos, Mata Atlântica, Unidade de Conservação

\section{Abstract}

Terrestrial mammals of Serra do Tabuleiro State Park, Santa Catarina, Brazil. Serra do Tabuleiro State Park, located in the central-eastern region of the state of Santa Catarina, in southern Brazil, has 85,000ha 
and a wide diversity of habitats (mangrove, restinga, dense and mixed rain forest, cloud forest and fields). From 1991 to 2010, 22 studies on its mammalian fauna were conducted, which used the following methods: live trapping (Young and double-door), pitfall traps, nesting boxes, mist nets, camera traps, direct observation and indirect evidence. Seventy-five autochthonous species (about 54\% of the terrestrial mammal species known from Santa Catarina), belonging to nine orders and 25 families, were recorded. Fifteen of these species are considered threatened at the state, national or global level. General aspects about the conservation of the mammals in the park, and the possibility of other species occurring in this area, are discussed.

Key words: Atlantic Rain Forest, Conservation, Conservation Unit, Mammals

\section{Introdução}

O Estado de Santa Catarina apresenta uma mastofauna diversificada, com 139 espécies de mamíferos terrestres distribuídas em 10 ordens (considerando-se Cingulata e Pilosa como ordens separadas) e 28 famílias, de acordo com os dados levantados por Cherem et al. (2004), complementados por registros recentes.

Para a conservação desta mastofauna, bem como de alguns outros componentes da diversidade biológica, a criação de áreas protegidas é uma das ferramentas mais importantes (GALINDO-LEAL; CÂMARA, 2005). No entanto, a delimitação espacial de uma área representa apenas o primeiro passo para sua proteção. Neste sentido, o inventário da biodiversidade é uma informação básica para a administração de unidades, permitindo a definição de prioridades de pesquisa e subsidiando a implantação de medidas de manejo (SCHNEIDER et al., 2000).

O Parque Estadual da Serra do Tabuleiro, localizado em uma área de Mata Atlântica no centro-leste do Estado de Santa Catarina, foi criado devido aos esforços do Dr. Raulino Reitz, com os objetivos de proteger os mananciais de água que abastecem os municípios da Grande Florianópolis; preservar espécies animais, vegetais e recursos paisagísticos ameaçados; e possibilitar a pesquisa, educação ambiental e turismo ecológico. É uma das maiores unidades de conservação do Estado e muito diversificada em tipos de ambientes, abrangendo inclusive algumas ilhas costeiras (KLEIN, 1981; ROSÁRIO, 2003; PEST, 2009).

Diversos estudos têm sido realizados com mamíferos nesse parque, entretanto, grande parte dessa informação permanece em relatórios e monografias ou trabalhos de conclusão de curso e poucos foram publicados (e.g., SALVADOR; FERNANDEZ, 2008a; 2008b; GOULART et al., 2009). Ainda, muitos dos nomes aplicados às espécies nesses documentos precisam ser corrigidos e atualizados, considerando-se as alterações recentes na nomenclatura mastozoológica.

Em vista disso, tem-se por objetivo apresentar as informações obtidas em 20 anos de estudos (1991 a 2010) sobre a diversidade e a conservação da mastofauna do Parque Estadual da Serra do Tabuleiro, na Mata Atlântica do Estado de Santa Catarina.

\section{Material e Métodos}

\section{Área de estudo}

O Parque Estadual da Serra do Tabuleiro -

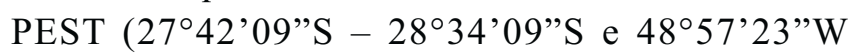
- 48 $43^{\prime}$ '09”'W; Figura 1) foi criado pelo Decreto N/ SETMA no 1260 de 01 de novembro de 1975. Sua área original de 87.405 hectares foi reduzida para 85.000 hectares com a aprovação da Lei Estadual no 347.3/08 (PEST, 2009). O PEST abrange os municípios de Águas Mornas, Florianópolis, Garopaba, Imaruí, Palhoça, Paulo Lopes, Santo Amaro da Imperatriz, São Bonifácio e São Martinho, no Estado de Santa Catarina, sul do Brasil. Sua sede localiza-se na Baixada do Maciambu, no município de Palhoça (ROSÁRIO, 2003).

O PEST abrange também 10 ilhas, as três ilhas do arquipélago de Moleques do Sul, as Três Irmãs (de Dentro, do Meio e de Fora), do Siriú, dos Corais, do Largo, dos Cardos e uma pequena parte da porção sul da Ilha de Santa Catarina.

O clima na maior parte do PEST, segundo o sistema de Koeppen, é do tipo Cfa, mesotérmico úmido com chuvas distribuídas uniformemente ao longo do ano e 


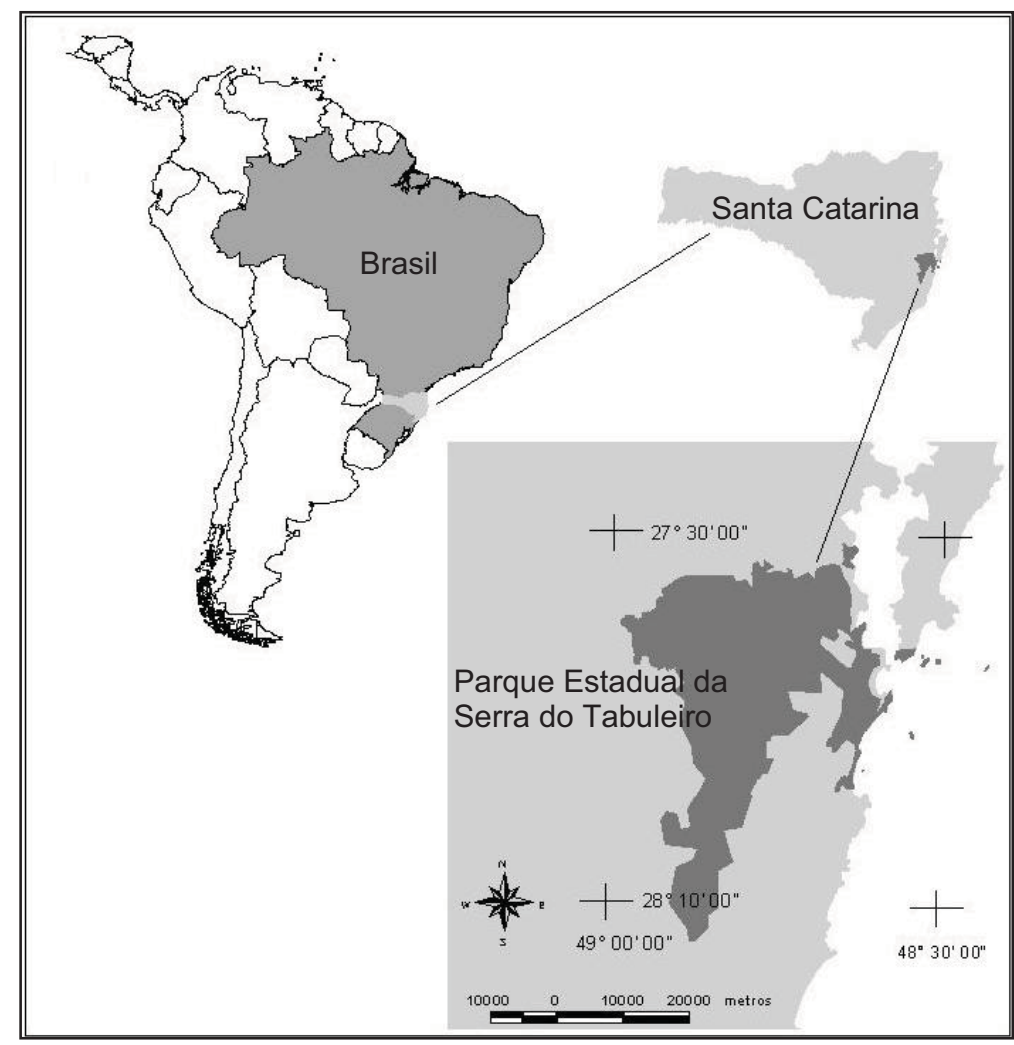

FIGURA 1: Localização do Parque Estadual da Serra do Tabuleiro no Estado de Santa Catarina, sul do Brasil. O mapa apresenta a área original do parque.

com verão quente (temperatura média do mês mais quente acima de $22^{\circ} \mathrm{C}$ ). Nas altitudes acima de 800 metros, constituídas pelas cristas das principais serras, o clima é do tipo $\mathrm{Cfb}$, mesotérmico úmido com verão fresco (temperatura média do mês mais quente abaixo de $22^{\circ} \mathrm{C}$ ).

O PEST apresenta uma grande diversidade de ambientes, incluindo formações pioneiras com influência marinha (restingas) e flúvio-marinha (manguezais), floresta ombrófila densa (manguezais e restingas), floresta ombrófila mista e estepe gramíneo-lenhosa, segundo nomenclatura empregada por IBGE (2004). A vegetação do parque é descrita detalhadamente por Klein (1981).

A amostragem de mamíferos ocorreu principalmente em três áreas na porção continental do parque e na ilha de Moleques do Sul. Outras áreas, incluindo as demais ilhas, foram vistoriadas eventualmente.

1. Hotel Plaza Caldas da Imperatriz (HPCI)

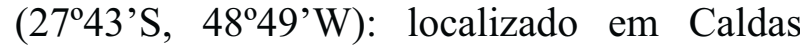

da Imperatriz, município de Santo Amaro da Imperatriz, tendo parte de sua área dentro do PEST, em floresta ombrófila densa (submontana a montana), em vários estágios de regeneração.

2. Baixada do Maciambu (BM) $\left(27^{\circ} 40^{\prime} \mathrm{S}\right.$, 4849’W): situa-se no município de Palhoça, onde está a sede do PEST. A vegetação local é composta por formação pioneira com influência marinha (restinga), ocorrendo desde campos sujos formados quase exclusivamente por herbáceas até vegetação arbustiva. Nas partes mais baixas ocorre vegetação brejosa.

3. São Bonifácio (SB) $\left(27^{\circ} 53^{\prime} \mathrm{S}, 48^{\circ} 51^{\prime} \mathrm{W}\right)$ : a terceira área de amostragem, no município de São Bonifácio, apresenta um mosaico de vegetação incluindo floresta ombrófila mista, estepe ombrófila (campos de altitude), campos higromórficos e pastagens.

4. Ilha de Moleques do Sul (IMS) (275' $45^{\prime}$ 'S, $\left.48^{\circ} 25^{\prime} 51^{\prime \prime} \mathrm{W}\right)$ : com 9,86 ha é a maior das ilhas 
do arquipélago de Moleques do Sul. Situa-se a $14 \mathrm{~km}$ do continente e a $8,25 \mathrm{~km}$ da ponta sudeste da Ilha de Santa Catarina. A vegetação na ilha é composta por formação pioneira com influência marinha (CHEREM et al., 1999; SALVADOR; FERNANDEZ, 2008a).

\section{Métodos}

Os trabalhos de campo no PEST tiveram início em janeiro de 1991 com a avaliação inicial de uma área florestal para um estudo de captura, marcação e recaptura de pequenos mamíferos não voadores próximo da área do HPCI, que foi realizado de abril de 1991 a abril de 1994. Desde então foram desenvolvidos outros 21 estudos com diferentes grupos de mamíferos e métodos diversificados (Tabela 1), além de terem sido obtidos registros casuais (vestígios, avistamentos e espécimens depositados em coleção).

Os métodos utilizados para amostragem da mastofauna incluíram:

a) Armadilhas de arame do tipo Young pequenas $(90 \times 150 \times 260 \mathrm{~mm})$ instaladas ao longo de transecções no solo, sub-bosque (amarradas a ripas de madeira pregadas em árvores a cerca de $2 \mathrm{~m}$ de altura) e dossel (adaptação do pulley method de MALCOLM, 1991; a uma altura média de 9,5m).

b) Armadilhas de arame do tipo Young médias $(180 \times 210 \times 400 \mathrm{~mm}$ e $200 \times 200 \times 600 \mathrm{~mm})$ instaladas em transecções no solo ou em locais selecionados, como carreiros.

c) Armadilhas de passagem $(210 \times 190 \times$ $960 \mathrm{~mm})$, com entrada dupla, instaladas em rios e córregos, conforme Bressiani e Graipel (2008).

d) Armadilhas de passagem $(110 \times 120 \mathrm{x}$ $590 \mathrm{~mm}$ ), com entrada dupla, sobre pontes artificiais construídas com bambus para captura de pequenos mamíferos, a uma altura entre 2 e $6 \mathrm{~m}$, seguindo Kierulff et al. (1991).

e) Armadilhas de interceptação e queda (pitfall-traps) com cerca guia de lona plástica de aproximadamente $60 \mathrm{~cm}$ de altura e recipientes de 30, 60 e $100 \mathrm{~L}$ em transecções de $100 \mathrm{~m}$, de acordo com Monteiro-Filho e Graipel (2006).

f) Redes-neblina de $7 \times 3 \mathrm{~m}$ para captura de quirópteros instaladas em dois pontos de amostragem no HPCI, durante 35 dias.

g) Caixas de nidificação confeccionadas com embalagens do tipo "Tetra Pak", de 16,5 x $9,5 \times 6,4 \mathrm{~cm}$ e volume de 1 litro,conforme Tortato e Campbell-Thompson (2006).

h) Armadilhas fotográficas analógicas e digitais (Modelo Tigrinus ${ }^{\circledR}$ ), posicionadas para registrar mamíferos no chão (GRAIPEL et al., 2008).

i) Armadilhas fotográficas analógicas e digitais (Modelo Tigrinus $\mathbb{R}$ ), posicionadas para registrar mamíferos no dossel (OLIVEIRA-SANTOS et al., 2008).

j) Registros diretos (carcaças e observação direta) e indiretos (pegadas, fezes e pelos) de mamíferos ao longo de trilhas.

Observação direta foi o único método empregado em nove ilhas que fazem parte do PEST. Apenas na ilha de Moleques do Sul foram utilizadas armadilhas Young para captura de exemplares de Cavia intermedia, utilizandose como isca milho apenas ou com frutas, beterraba ou cenoura. Nos outros estudos com armadilhas de arame foram utilizadas como iscas banana com ou sem pasta de amendoim, bacon ou peixe fresco. Em plataformas arbóreas associadas a armadilhas fotográficas foram utilizadas bananas.

As categorias de ameaça (vulnerável, em perigo ou criticamente em perigo) para as espécies sob risco de extinção foram obtidas em nível global (IUCN, 2010), para o território nacional (MMA, 2003) e para o Estado de Santa Catarina (IGNIS, 2010).

A nomenclatura dos mamíferos segue Gregorin (2006), Reis et al. (2006), Weksler et al. (2006), Voss e Jansa (2009) e Sicuro e Oliveira (2011). 
TABELA 1: Estudos desenvolvidos com mamíferos no Parque Estadual da Serra do Tabuleiro, Estado de Santa Catarina, sul do Brasil, de 1991 a 2010, indicando o número de espécies registradas e o esforço de amostragem por método. Número de armadilhas-noite para (a) Young no solo, (b) Young no subbosque e dossel, (c) armadilhas de passagem em rios e (d) sobre pontes de bambu, e (e) armadilhas de interceptação e queda. (f) Redes-neblina $\left(\mathrm{m}^{2} . h\right)$. Número de armadilhas-dia para (g) caixas de nidificação, (h) armadilhas fotográficas no chão e (i) no dossel. (j) Horas de observação direta. (k) Distância percorrida em trilhas (km).

\begin{tabular}{|c|c|c|c|c|c|c|c|c|c|c|c|c|c|c|}
\hline Pesquisadores & Período & Local* & $\begin{array}{l}\text { № } \\
\text { spp. }\end{array}$ & $\mathrm{a}$ & $\mathrm{b}$ & $\mathrm{c}$ & $d$ & e & $\mathrm{f}$ & $\mathrm{g}$ & $\mathrm{h}$ & $\mathrm{i}$ & $\mathrm{j}$ & $\mathrm{k}$ \\
\hline J. C. Voltolini; J. J. Cherem & IV/91 - IV/94 & HPCI & 16 & 7918 & 7883 & & & & & & & & & \\
\hline A. Filippini; C.H. Salvador & $2003-2009$ & 10 ilhas & 4 & & & & & & & & & & 96 & \\
\hline $\begin{array}{l}\text { J. Z. Matos; I. R. Ghizoni-Jr.; } \\
\text { S. L. Althoff } \\
\end{array}$ & III/94 - III/95 & HPCI & 13 & & & & & & 36960 & & & & & \\
\hline $\begin{array}{l}\text { R. R. Freitas; R. Illenseer; F. } \\
\text { Brüggemann; M. E. Graipel }\end{array}$ & $\mathrm{XII} / 96-\mathrm{VII} / 97$ & HPCI & 19 & & & & & & & & & & 256 & \\
\hline $\begin{array}{l}\text { R. Reinicke; P. C. Simões- } \\
\text { Lopes }\end{array}$ & $\mathrm{VI}-\mathrm{XI} / 99$ & BM & 4 & & & & & & & & & & 220 & \\
\hline $\begin{array}{l}\text { C. H. Salvador; M. E. Graipel; } \\
\text { P. C. Simões-Lopes }\end{array}$ & IX/00 - VI/01 & HPCI & 1 & 253 & & & & & & & & & & \\
\hline $\begin{array}{l}\text { A. Bevilacqua; M. A. Tortato; } \\
\text { P. C. Simões-Lopes }\end{array}$ & $\mathrm{X} / 01-\mathrm{VII} / 03$ & SB & 1 & & & & & & & & & & & 373 \\
\hline $\begin{array}{c}\text { K. Abati; M. P. Moraes; M. E. } \\
\text { Graipel }\end{array}$ & VIII/03 - V/04 & HPCI & 9 & & & & & 991 & & & & & & \\
\hline $\begin{array}{l}\text { M. P. Moraes; T. T. Moreira; } \\
\text { K. Abati; M. E. Graipel }\end{array}$ & $\mathrm{IX} / 03-\mathrm{X} / 04$ & HPCI & 10 & 840 & & & & 840 & & & & & & \\
\hline $\begin{array}{l}\text { T. T. Moreira; M. P. Moraes; } \\
\text { V. Kuhnen; M. E. Graipel }\end{array}$ & $\mathrm{XI} / 03-\mathrm{VII} / 05$ & HPCI & 11 & 6300 & & & & & & & & & & \\
\hline C. H. Salvador & $\mathrm{II} / 04-\mathrm{VII} / 05$ & IMS & 1 & 5542 & & & & & & & & & & \\
\hline $\begin{array}{l}\text { L. G. Oliveira-Santos; M. A. } \\
\text { Tortato }\end{array}$ & $\mathrm{III}-\mathrm{VI} / 05$ & $\mathrm{BM} / \mathrm{HPCI}$ & 9 & & & & & & & 228 & & 228 & & \\
\hline $\begin{array}{l}\text { F. Hoffmann; M. A. Tortato; } \\
\text { M. E. Graipel }\end{array}$ & IV $-\mathrm{XII} / 05$ & $\mathrm{BM}$ & 9 & & & & & & & & 57 & & & \\
\hline $\begin{array}{l}\text { L. G. R. Oliveira-Santos; F. } \\
\text { Goulart; M. A. Tortato; } \\
\text { I. R. Ghizoni-Jr.; M. E. Graipel }\end{array}$ & $\mathrm{XI} / 05-\mathrm{XI} / 06$ & HPCI & 10 & & & & & & & & 657 & & & \\
\hline $\begin{array}{c}\text { M. A. Tortato; L. G. R. } \\
\text { Oliveira-Santos; F. Goulart; } \\
\text { I. R. Ghizoni-Jr.; M. E. Graipel }\end{array}$ & $\mathrm{XI} / 05-\mathrm{XI} / 06$ & SB & 10 & & & & & & & & 1231 & & & \\
\hline $\begin{array}{l}\text { H. Mozerle; M. A. Tortato; C. } \\
\text { H. Salvador; J. J. Cherem }\end{array}$ & IX/06 - XI/07 & BM & 10 & 5625 & & & & 4800 & & & & & & \\
\hline N. Furnari & $\mathrm{XI} / 07-\mathrm{XII} / 09$ & IMS & 1 & 8127 & & & & & & & & & & \\
\hline $\begin{array}{l}\text { T. Maccarini; H. Mozerle; M. } \\
\text { E. Graipel }\end{array}$ & $\mathrm{I}-\mathrm{XII} / 08$ & HPCI & 2 & & & 1700 & & & & & & & & \\
\hline V. Kuhnen; M. E. Graipel & $\mathrm{VIII} / 08-\mathrm{X} / 09$ & HPCI & 15 & & & & & & & & 1570 & & & \\
\hline $\begin{array}{l}\text { R. Penedo-Ferreira; M. A. } \\
\text { Tortato }\end{array}$ & $\mathrm{XII} / 08-\mathrm{IV} / 10$ & HPCI & 2 & & & & & & & 2900 & & & & \\
\hline F. M. Fantacini; M. E. Graipel & $\mathrm{III} / 10-\mathrm{X} / 10$ & HPCI & 5 & & & & 2000 & & & & & & & \\
\hline $\begin{array}{l}\text { D. Dias; B. P. Vieira; M. E. } \\
\text { Graipel }\end{array}$ & $\mathrm{X} / 10 * *$ & $\mathrm{BM} / \mathrm{HPCI}$ & 8 & & & & & 138 & & & & & & \\
\hline TOTAL & $\mathrm{IV} / 01-\mathrm{X} / 10$ & & 75 & 34605 & 7833 & 1700 & 2000 & 6769 & 36960 & 3128 & 3515 & 228 & 1012 & 373 \\
\hline
\end{tabular}

* BM = Baixada do Maciambu; HPCI = Hotel Plaza Caldas da Imperatriz; IMS = Ilha de Moleques do Sul; SB = São Bonifácio. ** estudo em andamento. 


\section{Resultados}

Foram registradas 75 espécies de mamíferos autóctones no PEST pertencentes a nove ordens: Rodentia (26 espécies), Chiroptera (16), Carnivora (12), Didelphimorphia (10), Cingulata (4), Artiodactyla (3), Primates (2), Pilosa (1) e Perissodactyla (1). Com relação às famílias, o maior número de espécies foi registrado para Cricetidae (16), seguida por Phyllostomidae (11) e Didelphidae (10) (Tabelas 1 e 2; Anexo 1).

Além destas, também foram registradas 12 espécies alóctones na porção continental do PEST, incluindo: Callithrix sp. (sagui), Canis familiaris (cachorro-doméstico), Felis catus (gato-doméstico), Equus caballus (cavalo), Sus scrofa (porcos ferais), Bos taurus (boi), Capra hircus (cabra), Ovis aries (ovelha), Lepus europaeus (lebre-europeia), Mus musculus (camundongo-doméstico), Rattus norvegicus (ratazana) e $R$. rattus (rato-preto).

Em relação às ilhas protegidas pelo PEST, foram registradas as seguintes espécies autóctones: $C$. intermedia (preá) na ilha de Moleques do Sul; Lontra longicaudis (lontra) nas ilhas Irmã de Dentro, Irmã de Fora e Siriú; e Didelphis aurita (gambá) nas ilhas Irmã do Meio, dos Corais e do Largo. Duas espécies alóctones foram também encontradas: Cavia porcellus (porquinho-da-Índia) na Ilha dos Cardos e C. hircus (cabra) na Ilha dos Corais.

Quinze mamíferos do PEST integram pelo menos uma das listas de espécies ameaçadas: três espécies em nível global (um carnívoro Felidae, um perissodáctilo e um roedor), cinco em nível nacional (um quiróptero e quatro carnívoros Felidae) e 12 em nível estadual (dois marsupiais Didelphimorphia, dois quirópteros, um primata, dois carnívoros Felidae, um perissodáctilo, dois artiodáctilos e dois roedores) (Tabela 2).

O esforço diferencial de amostragem no PEST não permite uma comparação mais detalhada entre as áreas e ambientes. No entanto, algumas espécies foram registradas apenas ou principalmente associadas às áreas baixas na planície litorânea, como Lutreolina crassicaudata, Noctilio leporinus, Holochilus sp. e Cavia magna.

TABELA 2: Relação dos mamíferos autóctones registrados no Parque Estadual da Serra do Tabuleiro, Estado de Santa Catarina, sul do Brasil, de 1991 a 2010.

\begin{tabular}{|c|c|c|c|c|c|}
\hline Táxon & Nome Comum & Métodoa & Ambiente $^{b}$ & Local $^{c}$ & Status $^{d}$ \\
\hline \multicolumn{6}{|l|}{ DIDELPHIMORPHIA } \\
\hline \multicolumn{6}{|l|}{ Didelphidae } \\
\hline Chironectes minimus (Zimmermann, 1780) & cuíca-d’água & $\mathrm{C} / \mathrm{O} / \mathrm{P}$ & $\mathrm{FE} / \mathrm{MC}$ & HPCI & $\mathrm{SC}-\mathrm{VU}$ \\
\hline Cryptonanus sp. & guaiquica & $\mathrm{C}$ & $\mathrm{RE}$ & $\mathrm{BM}$ & \\
\hline Didelphis albiventris Lund, 1840 & gambá-de-orelha-branca & $\mathrm{AF} / \mathrm{C} / \mathrm{O}$ & $\mathrm{FA} / \mathrm{FE} / \mathrm{MC} / \mathrm{RE}$ & $\mathrm{BM} / \mathrm{HPCI} / \mathrm{SB}$ & \\
\hline Didelphis aurita Wied-Neuwied, 1826 & gambá-de-orelha-preta & $\mathrm{AF} / \mathrm{C} / \mathrm{O}$ & $\mathrm{AI} / \mathrm{FE} / \mathrm{MC} / \mathrm{RE}$ & BM/HPCI/ILH & \\
\hline Gracilinanus microtarsus (Wagner, 1842) & guaiquica & $\mathrm{C}$ & $\mathrm{FE}$ & HPCI & \\
\hline Lutreolina crassicaudata (Desmarest, 1804) & cuíca & $\mathrm{O}$ & $\mathrm{RE}$ & $\mathrm{BM}$ & $\mathrm{SC}-\mathrm{VU}$ \\
\hline Marmosa paraguayana (Tate, 1931) & guaiquica & $\mathrm{AF} / \mathrm{C}$ & $\mathrm{FE} / \mathrm{MC} / \mathrm{RE}$ & $\mathrm{BM} / \mathrm{HPCI}$ & \\
\hline Monodelphis iheringi (Thomas, 1888) & cuíca & $\mathrm{C}$ & $\mathrm{FE} / \mathrm{RE}$ & BM HPCI & \\
\hline Monodelphis scalops (Thomas, 1888) & cuíca & $\mathrm{C}$ & $\mathrm{FE}$ & HPCI & \\
\hline Philander frenatus (Linnaeus, 1758) & cuíca & $\mathrm{AF} / \mathrm{C}$ & $\mathrm{FE} / \mathrm{MC}$ & HPCI & \\
\hline \multicolumn{6}{|l|}{ CINGULATA } \\
\hline \multicolumn{6}{|l|}{ Dasypodidae } \\
\hline Cabassous tatouay (Desmarest, 1804) & tatu-de-rabo-mole & $\mathrm{AF} / \mathrm{P} / \mathrm{O}$ & FE & HPCI & \\
\hline Dasypus novemcinctus Linnaeus, 1758 & tatu-galinha & $\mathrm{AF} / \mathrm{O} / \mathrm{P} / \uparrow$ & $\mathrm{FE} / \mathrm{RE}$ & $\mathrm{BM} / \mathrm{HPCI}$ & \\
\hline Dasypus septemcinctus Linnaeus, 1758 & tatu-mulita & $\mathrm{O} / \mathrm{P}$ & FE & HPCI & \\
\hline Euphractus sexcinctus (Linnaeus, 1758) & tatu-peludo & $\mathrm{P}$ & FE & HPCI & \\
\hline \multicolumn{6}{|l|}{ PILOSA } \\
\hline \multicolumn{6}{|l|}{ Myrmecophagidae } \\
\hline Tamandua tetradactyla (Linnaeus, 1758) & tamanduá-mirim & $\mathrm{AF} / \uparrow$ & $\mathrm{FE} / \mathrm{RE}$ & $\mathrm{BM} / \mathrm{HPCI}$ & \\
\hline \multicolumn{6}{|l|}{ CHIROPTERA } \\
\hline Noctilionidae & & & & & \\
\hline
\end{tabular}




\begin{tabular}{|c|c|c|c|c|c|}
\hline Táxon & Nome Comum & Método ${ }^{\mathrm{a}}$ & Ambiente $^{\mathrm{b}}$ & Local $^{\mathrm{c}}$ & Status $^{d}$ \\
\hline $\begin{array}{l}\text { Noctilio leporinus (Linnaeus, 1758) } \\
\text { Phyllostomidae }\end{array}$ & morcego-pescador & $\mathrm{O}$ & $\mathrm{RE}$ & $\mathrm{BM}$ & \\
\hline Artibeus fimbriatus Gray, 1838 & morcego & $\mathrm{C}$ & $\mathrm{FE}$ & HPCI & \\
\hline Artibeus lituratus (Olfers, 1818) & morcego & $\mathrm{C}$ & $\mathrm{FE}$ & HPCI & \\
\hline Artibeus obscurus Schinz, 1821 & morcego & $\mathrm{C}$ & FE & HPCI & \\
\hline Carollia perspicillata (Linnaeus,1758) & morcego & $\mathrm{C}$ & $\mathrm{FE}$ & HPCI & \\
\hline Diphylla ecaudata Spix, 1823 & morcego & $\mathrm{C}$ & $\mathrm{FE}$ & HPCI & SC-EN \\
\hline Mimon bennettii (Gray, 1838) & morcego & $\mathrm{C}$ & $\mathrm{FE}$ & HPCI & \\
\hline Platyrrhinus lineatus (E. Geoffroy, 1810) & morcego & $\mathrm{C}$ & $\mathrm{FE}$ & HPCI & \\
\hline Pygoderma bilabiatum (Wagner, 1843) & morcego & $\mathrm{C}$ & $\mathrm{FE}$ & HPCI & \\
\hline Sturnira lilium (E. Geoffroy, 1810) & morcego & $\mathrm{AF} / \mathrm{C}$ & $\mathrm{FE}$ & HPCI & \\
\hline Sturnira tildae de la Torre, 1959 & morcego & $\mathrm{C}$ & FE & HPCI & $\mathrm{SC}-\mathrm{VU}$ \\
\hline $\begin{array}{l}\text { Vampyressa pusilla (Wagner, 1843) } \\
\text { Vespertilionidae }\end{array}$ & morcego & $\mathrm{C}$ & $\mathrm{FE}$ & HPCI & \\
\hline Eptesicus brasiliensis (Desmarest, 1819) & morcego & $\mathrm{C}$ & FE & HPCI & \\
\hline $\begin{array}{l}\text { Myotis ruber (E. Geoffroy, 1806) } \\
\text { Molossidae }\end{array}$ & morcego & $\mathrm{C}$ & $\mathrm{FE}$ & HPCI & BR-VU \\
\hline Molossus molossus (Pallas, 1766) & morcego & $\mathrm{C}$ & FE & HPCI & \\
\hline $\begin{array}{l}\text { Tadarida brasiliensis (I. Geoffroy, 1824) } \\
\text { PRIMATES } \\
\text { Atelidae }\end{array}$ & morcego & $\mathrm{C}$ & FE & HPCI & \\
\hline $\begin{array}{l}\text { Alouatta clamitans Cabrera, } 1940 \\
\text { Cebidae }\end{array}$ & bugio & $\mathrm{O} / \uparrow$ & $\mathrm{FE}$ & HPCI & SC-VU \\
\hline $\begin{array}{l}\text { Cebus nigritus (Goldfuss, 1809) } \\
\text { CARNIVORA } \\
\text { Canidae }\end{array}$ & mico, macaco-prego & $\mathrm{O}$ & $\mathrm{FE}$ & HPCI & \\
\hline Cerdocyon thous (Linnaeus, 1766) & $\begin{array}{l}\text { graxaim, cachorro-do- } \\
\text { mato }\end{array}$ & $\begin{array}{l}\mathrm{AF} / \mathrm{F} / \\
\mathrm{O} / \mathrm{P} / \uparrow\end{array}$ & $\mathrm{FE} / \mathrm{RE}$ & $\mathrm{BM} / \mathrm{HPCI}$ & \\
\hline $\begin{array}{l}\text { Felidae } \\
\text { Herpailurus yagouaroundi (É. Geoffroy, } \\
\text { 1803) }\end{array}$ & $\begin{array}{l}\text { gato-mourisco, } \\
\text { jaguarundi }\end{array}$ & $\mathrm{AF} / \mathrm{O} / \mathrm{P}$ & FE & HPCI & \\
\hline Leopardus pardalis (Linnaeus, 1758) & jaguatirica & $\mathrm{AF}$ & FE & HPCI & $\begin{array}{l}\text { BR-VU / } \\
\text { SC-EN }\end{array}$ \\
\hline Leopardus tigrinus (Schreber, 1775) & gato-do-mato, oncinha & $\mathrm{AF} / \dagger$ & $\mathrm{FE} / \mathrm{RE}$ & $\mathrm{BM} / \mathrm{HPCI}$ & $\begin{array}{c}\text { IU-VU / BR- } \\
\text { VU }\end{array}$ \\
\hline Leopardus wiedii (Schinz, 1821) & gato-do-mato, oncinha & $\mathrm{AF} / \uparrow$ & $\mathrm{FE}$ & HPCI & BR-VU \\
\hline Puma concolor (Linnaeus, 1771) & puma, leão-baio & $\mathrm{AF} / \mathrm{F} / \mathrm{P}$ & $\mathrm{CA} / \mathrm{FE}$ & $\mathrm{HPCI} / \mathrm{SB}$ & $\begin{array}{l}\text { BR-VU / } \\
\text { SC-VU }\end{array}$ \\
\hline $\begin{array}{l}\text { Mephitidae } \\
\text { Conepatus chinga (Molina, 1782) } \\
\text { Mustelidae }\end{array}$ & zorrilho & $\mathrm{P}$ & CA & SB & \\
\hline $\begin{array}{l}\text { Lontra longicaudis (Olfers, 1818) } \\
\text { Eira barbara (Linnaeus, 1758) } \\
\text { Galictis cuja (Molina, 1782) } \\
\quad \text { Procyonidae }\end{array}$ & $\begin{array}{l}\text { lontra } \\
\text { irara, papa-mel } \\
\text { furão }\end{array}$ & $\begin{array}{c}\mathrm{O} \\
\mathrm{AF} / \mathrm{O} \\
\mathrm{AF} / \mathrm{O}\end{array}$ & $\begin{array}{l}\mathrm{AI} / \mathrm{MC} / \mathrm{RE} \\
\quad \mathrm{FE} \\
\mathrm{FA} / \mathrm{FE} / \mathrm{RE}\end{array}$ & $\begin{array}{c}\mathrm{BM} / \mathrm{ILH} \\
\mathrm{HPCI} \\
\mathrm{BM} / \mathrm{HPCI} / \mathrm{SB}\end{array}$ & \\
\hline $\begin{array}{l}\text { Nasua nasua (Linnaeus, 1766) } \\
\text { Procyon cancrivorus (G. Cuvier, 1798) } \\
\text { PERISSODACTYLA } \\
\text { Tapiridae }\end{array}$ & $\begin{array}{c}\text { quati } \\
\text { mão-pelada, guaxinim }\end{array}$ & $\begin{array}{l}\mathrm{AF} / \mathrm{C} / \mathrm{O} \\
\mathrm{AF} / \mathrm{P}\end{array}$ & $\begin{array}{c}\mathrm{FA} / \mathrm{FE} \\
\mathrm{FE} / \mathrm{MC} / \mathrm{RE}\end{array}$ & $\begin{array}{l}\mathrm{HPCI} / \mathrm{SB} \\
\mathrm{BM} / \mathrm{HPCI}\end{array}$ & \\
\hline $\begin{array}{l}\text { Tapirus terrestris (Linnaeus, 1758) } \\
\text { ARTIODACTYLA } \\
\text { Tayassuidae }\end{array}$ & anta & $\mathrm{AF} / \mathrm{F} / \mathrm{P} / \mathrm{O}$ & $\mathrm{CA} / \mathrm{FA} / \mathrm{FE} / \mathrm{RE}$ & $\mathrm{BM} / \mathrm{HPCI} / \mathrm{SB}$ & $\begin{array}{l}\text { IU-VU / SC- } \\
\text { EN }\end{array}$ \\
\hline
\end{tabular}




\begin{tabular}{|c|c|c|c|c|c|}
\hline Táxon & Nome Comum & Método $^{\mathrm{a}}$ & Ambiente $^{\mathrm{b}}$ & Local $^{\mathrm{c}}$ & Status $^{\mathrm{d}}$ \\
\hline Pecari tajacu (Linnaeus, 1758) & cateto, porco-do-mato & $\mathrm{AF} / \mathrm{P}$ & FA & SB & SC-VU \\
\hline $\begin{array}{l}\text { Tayassu pecari (Link, 1795) } \\
\quad \text { Cervidae }\end{array}$ & queixada, porco-do-mato & $\mathrm{AF}$ & FA & SB & SC-CR \\
\hline $\begin{array}{l}\text { Mazama gouazoubira }(\mathrm{G} \text {. Fischer, 1814) } \\
\text { RODENTIA } \\
\text { Sciuridae }\end{array}$ & veado catingueiro & $\mathrm{AF}$ & FE & HPCI & \\
\hline $\begin{array}{l}\text { Guerlinguetus ingrami (Thomas, 1901) } \\
\text { Cricetidae }\end{array}$ & esquilo, serelepe & $\mathrm{O}$ & FE & HPCI & \\
\hline Abrawayaomys ruschii Cunha e Cruz, 1979 & rato-de-espinho & $\mathrm{C}$ & $\mathrm{FE}$ & HPCI & \\
\hline Akodon montensis Thomas, 1913 & rato-do-mato & $\mathrm{C}$ & $\mathrm{FE} / \mathrm{MC} / \mathrm{RE}$ & $\mathrm{BM} / \mathrm{HPCI}$ & \\
\hline Brucepattersonius iheringi (Thomas, 1896) & rato-do-mato & $\mathrm{C}$ & $\mathrm{FE}$ & HPCI & \\
\hline Delomys sublineatus (Thomas, 1903) & rato-do-mato & $\mathrm{C}$ & $\mathrm{FE} / \mathrm{MC}$ & HPCI & \\
\hline $\begin{array}{l}\text { Drymoreomys albimaculatus Percequillo, } \\
\text { Weksler e Costa, } 2011\end{array}$ & rato-do-mato & $\mathrm{C}$ & FE & HPCI & \\
\hline Euryoryzomys russatus (Wagner, 1848) & rato-do-mato & $\mathrm{C}$ & FE & HPCI & \\
\hline Holochilus sp. & rato-do-banhado & $\mathrm{C}$ & $\mathrm{RE}$ & $\mathrm{BM}$ & \\
\hline Juliomys pictipes (Osgood, 1933) & rato-do-mato & $\mathrm{AF} / \mathrm{C}$ & $\mathrm{FE}$ & HPCI & \\
\hline Nectomys squamipes (Brants, 1827) & rato-do-mato & $\mathrm{C}$ & $\mathrm{FE} / \mathrm{MC} / \mathrm{RE}$ & $\mathrm{BM} / \mathrm{HPCI}$ & \\
\hline Oligoryzomys flavescens (Waterhouse, 1837) & rato-do-mato & $\mathrm{C}$ & $\mathrm{RE}$ & $\mathrm{BM}$ & \\
\hline Oligoryzomys nigripes (Olfers, 1818) & rato-do-mato & $\mathrm{C}$ & $\mathrm{FE} / \mathrm{RE}$ & $\mathrm{BM} / \mathrm{HPCI}$ & \\
\hline Oxymycterus judex Thomas, 1909 & rato-do-mato & $\mathrm{C}$ & $\mathrm{FE}$ & HPCI & \\
\hline Rhipidomys mastacalis (Lund, 1840) ${ }^{\mathrm{e}}$ & rato-do-mato & $\mathrm{C}$ & $\mathrm{FE}$ & HPCI & \\
\hline Sooretamys angouya (G. Fischer, 1814) & rato-do-mato & $\mathrm{AF} / \mathrm{C}$ & $\mathrm{FE} / \mathrm{RE}$ & $\mathrm{BM} / \mathrm{HPCI}$ & \\
\hline Thaptomys nigrita (Lichtenstein, 1829) & rato-do-mato & $\mathrm{C}$ & $\mathrm{FE}$ & HPCI & \\
\hline $\begin{array}{l}\text { gen. e sp. indet. } \\
\text { Erethizontidae }\end{array}$ & rato-do-mato & $\mathrm{C}$ & $\mathrm{FE}$ & HPCI & \\
\hline $\begin{array}{l}\text { Sphiggurus villosus (F. Cuvier, 1823) } \\
\text { Caviidae }\end{array}$ & ouriço & $\mathrm{O}$ & $\mathrm{FE} / \mathrm{RE}$ & $\mathrm{BM} / \mathrm{HPCI}$ & \\
\hline $\begin{array}{l}\text { Cavia intermedia Cherem, Olimpio e } \\
\text { Ximenez, } 1999\end{array}$ & preá & $\mathrm{C}$ & AI & IMS & $\begin{array}{l}\mathrm{IU}-\mathrm{CR} / \mathrm{SC}- \\
\quad \mathrm{CR}\end{array}$ \\
\hline Cavia magna Ximenez, 1980 & preá & $\mathrm{C}$ & $\mathrm{RE}$ & $\mathrm{BM}$ & \\
\hline $\begin{array}{l}\text { Hydrochoerus hydrochaeris (Linnaeus, 1766) } \\
\text { Dasyproctidae }\end{array}$ & capivara & $\mathrm{AF} / \mathrm{F} / \mathrm{O} / \mathrm{P}$ & RE & $\mathrm{BM}$ & \\
\hline $\begin{array}{l}\text { Dasyprocta azarae Lichtenstein, } 1823 \\
\text { Cuniculidae }\end{array}$ & cutia & $\mathrm{AF} / \mathrm{O} / \mathrm{P}$ & $\mathrm{FE} / \mathrm{RE}$ & $\mathrm{BM} / \mathrm{HPCI}$ & \\
\hline $\begin{array}{l}\text { Cuniculus paca (Linnaeus, 1766) } \\
\text { Echimyidae }\end{array}$ & paca & $\mathrm{P}$ & $\mathrm{FE}$ & HPCI & $\mathrm{SC}-\mathrm{VU}$ \\
\hline $\begin{array}{l}\text { Phyllomys sulinus Leite, Christoff e } \\
\text { Fagundes, } 2008\end{array}$ & rato-de-espinho & $\mathrm{C}$ & $\mathrm{FE}$ & HPCI & \\
\hline $\begin{array}{l}\text { Euryzygomatomys spinosus (G. Fischer, 1814) } \\
\text { Myocastoridae }\end{array}$ & rato-de espinho & $\mathrm{C}$ & $\mathrm{FE} / \mathrm{MC}$ & HPCI & \\
\hline Myocastor coypus (Molina, 1782) & ratão-do-banhado & $\mathrm{AF} / \mathrm{F} / \mathrm{O} / \mathrm{P}$ & $\mathrm{RE}$ & $\mathrm{BM}$ & \\
\hline
\end{tabular}

${ }^{a}$ Método: $\mathrm{AF}$ = armadilha-fotográfica; $\mathrm{C}$ = captura (armadilhas Young, de dupla entrada, interceptação e queda, redes-neblina ou caixas de nidificação); $\mathrm{F}=$ fezes; $\mathrm{O}=$ observação direta; $\mathrm{P}=$ pegadas; $\dagger=$ encontrado morto.

${ }^{\mathrm{b}}$ Ambiente: $\mathrm{AI}$ = formação pioneira com influência marinha em ambiente insular; $\mathrm{CA}=$ estepe ombrófila (campos de altitude); FA = floresta ombrófila mista (floresta com araucárias); $\mathrm{FE}=$ floresta ombrófila densa de encosta; $\mathrm{MC}=$ mata ciliar; $\mathrm{RE}=$ formação pioneira com influência marinha (restinga).

${ }^{\mathrm{c}}$ Local: $\mathrm{BM}=$ Baixada do Maciambu; HPCI = Hotel Plaza Caldas da Imperatriz; IMS = Ilha de Moleques do Sul; ILH = outras ilhas; SB $=$ São Bonifácio.

d Status de conservação: IU = espécie ameaçada globalmente (IUCN, 2010); BR = ameaçada no território nacional (MMA, 2003); SC = ameaçada para Santa Catarina (IGNIS, 2010). VU = vulnerável; EN = em perigo; $\mathrm{CR}=$ criticamente em perigo.

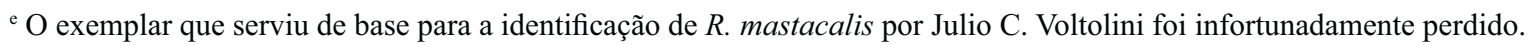




\section{Discussão}

O número de espécies de mamíferos terrestres autóctones registrados no PEST (75) representa cerca de $54 \%$ do total conhecido (excluindo pinípedes e cetáceos) para Santa Catarina (J. J. CHEREM, dados não publicados). Esse é um número elevado em comparação com os dados publicados para outras unidades de conservação brasileiras. Por exemplo, no Parque Nacional do Itatiaia e na Reserva Biológica de Poço das Antas, Rio de Janeiro, foram listadas 86 e 77 espécies, respectivamente (BRITO et al., 2004; GEISE et al., 2004; DIAS et al., 2010; MARTINS et al., 2010); na Estação Ecológica de Jataí, São Paulo, 72 espécies (GARGAGLIONI et al., 1998); no Parque Florestal Estadual do Rio Doce e no Parque Nacional da Serra da Canastra, Minas Gerais, 60 e 59 espécies, respectivamente (STALLINGS et al., 1991; SCHNEIDER et al., 2000); no Parque Estadual do Desengano, Rio de Janeiro, 56 espécies (MODESTO et al., 2008); e na RPPN Volta Velha, Santa Catarina, 47 espécies (QUADROS; CÁCERES, 2001). No entanto, deve-se considerar que, além da influência de fatores bióticos e abióticos em cada unidade, como dimensões, diversidade de ambientes e características do entorno, os métodos e os esforços de amostragem utilizados são diferentes.

A importância do PEST para a mastofauna também é expressa no número de espécies ameaçadas de extinção, 15, se consideradas as listas global (IUCN, 2010), nacional (MMA, 2003) e estadual (IGNIS, 2010), incluindo dois táxons criticamente em perigo, Tayassu pecari (queixada) e C. intermedia (preá, endêmico da ilha de Moleques do Sul). Na lista estadual, que contém 29 espécies de mamíferos terrestres, 12 (41\%) foram registradas no PEST, incluindo espécies que sofrem intensa pressão de caça (alto valor cinegético), como a anta, o queixada e o cateto.

Destaca-se também o registro de Drymoreomys albimaculatus, pequeno roedor cricetídeo descrito recentemente (PERCEQUILLO et al., 2011), cujos parátipos incluem um exemplar coletado no PEST e outros no Parque Natural Municipal Nascentes do Garcia, no município de Blumenau. Outros táxons pouco conhecidos, como Cryptonanus sp., Holochilus sp. e outro pequeno roedor não identificado, também foram levantados para o PEST e a determinação da espécie a que pertencem requer estudos mais aprofundados.

O esforço contínuo de amostragem e em diferentes ambientes é um fator relevante em inventários faunísticos (VOSS; EMMONS, 1996). Cryptonanus sp., Holochilus sp. e Oligoryzomys flavescens foram registrados no PEST somente a partir de 2006 (16o ano de estudo), quando a Baixada do Maciambu foi amostrada com armadilhas de arame e de interceptação e queda. Leopardus pardalis possuía registro para São Bonifácio (mas não é certo se dentro dos limites do parque), em 1973, sendo novamente registrado apenas em 2009 por armadilha fotográfica no HPCI (KUHNEN et al., 2011).

Isto indica que outras espécies podem vir a ser encontradas no PEST, como os roedores Akodon serrensis Thomas, 1902, Oxymycterus nasutus (Waterhouse, 1837), Scapteromys sp. e Kannabateomys amblyonyx (Wagner, 1845), com base na distribuição conhecida ou esperada para essas espécies (CHEREM et al., 2004).

Os quirópteros são o grupo menos amostrado entre os mamíferos, tendo sido realizado apenas um estudo no PEST. Anoura caudifera, A. geoffroyi, Artibeus jamaicensis, Desmodus rotundus, Dasypterus ega, Eptesicus diminutus, E. furinalis, Lasiurus borealis, Myotis levis, M. nigricans, M. riparius e Nyctinomops laticaudatus são conhecidos para o entorno (CHEREM et al., 2004) e devem ocorrer no parque.

Para os demais grupos de mamíferos, a possibilidade de novos registros de espécies no PEST é menor, mas podem ocorrer, por exemplo, Monodelphis americana (Müller, 1776), Lycalopex gymnocercus (G. Fischer, 1814), Mazama nana (Hensel, 1872) e Sylvilagus brasiliensis (Linnaeus, 1758). Reitz et al. (1982) citaram Ozotoceros bezoarticus (Linnaeus, 1758) (veadocampeiro) como uma espécie rara no PEST e que um exemplar de Mazama americana (Erxleben, 1777) foi introduzido neste parque como parte do Projeto de "Restauração da Fauna Desaparecida na Baixada do Maciambu", iniciado em junho de 1978, mas não se dispõe de registros recentes dessas espécies. Reitz et al. (1982) citaram ainda que Blastocerus dichotomus 
(Illiger, 1815) (cervo-do-pantanal) e Myrmecophaga tridactyla Linnaeus, 1758 (tamanduá-bandeira) já tinham sido extintos no PEST. Estes dois mamíferos não têm registros atuais no Estado de Santa Catarina (CHEREM et al., 2004). Panthera onca (Linnaeus, 1758) (onçapintada) também é considerada extinta localmente.

Apesar de espécies de mamíferos de grande porte terem sido eliminadas pela caça e, em menor grau pela redução e fragmentação do habitat natural, a extensão relativamente grande do PEST ainda garante a ocorrência de espécies ameaçadas e de alto interesse cinegético, como Tapirus terrestris (anta) e T. pecari (queixada), bem como mamíferos de pequeno porte possivelmente raros naturalmente, como Abrawayaomys ruschii.

Por outro lado, a pressão antrópica sobre o parque e seu entorno é evidenciada pela escassez de registros de L. pardalis (jaguatirica), espécie que deveria ser encontrada com facilidade em grandes áreas florestais com o uso de armadilhas fotográficas, como ocorre na RPPN Caraguatá, a cerca de $40 \mathrm{~km}$ ao norte do PEST (GOULART et al., 2009). Além disso, o PEST e seu entorno vêm sofrendo diversas ameaças à integridade de sua fauna como a presença de espécies alóctones (domésticas ou invasoras); mudanças de usos do solo, como conversão de ambientes naturais, expansão imobiliária, drenagem das restingas e florestas das terras baixas; caça; desmatamento e retirada ilegal de produtos florestais (FATMA, 2005). Além disso, práticas de manejo tidas como benéficas há algum tempo podem ter consequências indesejáveis, como doenças, alteração da comunidade local, etc., decorrentes da introdução de espécies de procedência desconhecida ou de regiões distantes, como no caso de exemplares de Myocastor coypus (ratão-do-banhado) oriundos do estado do Rio Grande do Sul e de T. terrestris (anta) provenientes do norte do Brasil introduzidos no parque. Estes impactos, associados à diminuição da área do PEST com a aprovação da Lei Estadual 347.3/08 e a proposta de alteração do código florestal que reduziria as Áreas de Proteção Permanente e Reserva Legal no entorno do parque, demonstram a fragilidade a que estão sujeitas unidades de conservação estabelecidas no Brasil.

\section{Agradecimentos}

Aos inúmeros alunos e biólogos que auxiliaram nos projetos ao longo desses 20 anos de pesquisa no PEST e a Nilton Cáceres e Marcio Soldateli pela revisão do manuscrito. Esses projetos contaram com o apoio logístico e/ou financeiro de várias instituições, como o Hotel Plaza Caldas da Imperatriz, o Programa Funpesquisa da UFSC, a Fundação O Boticário, a Fapesp e a ONG Conservação Internacional do Brasil.

\section{Referências}

BRESSIANI, V. B.; GRAIPEL, M. E. Comparação de métodos para captura da cuíca-d'água, Chironectes minimus (Zimmerman, 1780) (Mammalia, Didelphidae) no sul do Brasil. Mastozoologia Neotropical, Mendoza, v. 15, n. 1, p. 33-39, 2008.

BRITO, D.; OLIVEIRA L. C.; MELLO, M. A. R. An overview of mammalian conservation at Poço das Antas Biological Reserve, southeastern Brazil. Journal for Nature Conservation, Tilburg, v. 12, p. 219-228, 2004.

CHEREM, J. J.; ALTHOFF, S. L.; SIMÕES-LOPES, P. C.; GRAIPEL, M. E. Lista dos mamíferos do estado de Santa Catarina, sul do Brasil. Mastozoologia Neotropical, Mendoza, v. 11, n. 2, p. 151-184, 2004.

CHEREM, J. J.; OLIMPIO, J.; XIMENEZ, A. Descrição de uma nova espécie do gênero Cavia Pallas, 1766 (Mammalia - Caviidae) das Ilhas dos Moleques do Sul, Santa Catarina, Sul do Brasil. Biotemas, Florianópolis, v. 12, n. 1, p. 95-117, 1999.

DIAS, D.; PEREIRA, S. N.; MAAS, A. C. S.; MARTINS, M. A.; BOLZAN, D. P.; PERACCHI, A. L. Quirópteros das regiões Centro-Sul e Médio Paraíba do estado do Rio de Janeiro (Mammalia, Chiroptera). Chiroptera Neotropical, Brasília, v. 16, p. 579-585, 2010.

FATMA - FUNDAÇÃO DO MEIO AMBIENTE. Parque Estadual da Serra do Tabuleiro em Santa Catarina. Florianópolis: FATMA, 2005. Disponível em: <http://www.fatma.gov.br/projetos/ convenio_microbaciasII.htm>. Acesso em: 1 mar. 2005.

GALINDO-LEAL, C.; CÂMARA, I. G. Mata Atlântica: biodiversidade, ameaças e perspectivas. Belo Horizonte: Fundação SOS Mata Atlântica, 2005. 471 p.

GARGAGLIONI, L. H.; BATALHÃO, M. E.; LAPENTA, M. J.; CARVALHO, M. F.; ROSSI, R. V.; VERULI, V. P. Mamíferos da Estação Ecológica de Jataí, Luiz Antônio, São Paulo. Papéis Avulsos de Zoologia, São Paulo, v. 40, n. 17, p. 267-287, 1998.

GEISE, L.; PEREIRA, L. G.; BOSSI, D. E. P.; BERGALLO, H. G. Pattern of elevational distribution and richness of non volant mammals in Itatiaia National Park and its surroundings, in southestern Brazil. Brazilian Journal of Biology, São Carlos, v. 64, p. 599-612, 2004.

GOULART, F. V .B.; CÁCERES, N. C.; GRAIPEL, M. E.; TORTATO, M. A.; GHIZONI-JR., I. R.; OLIVEIRA-SANTOS, L. G. R. Habitat selection by large mammals in southern Brazilian 
Atlantic Forest. Mammalian Biology, Jena, v. 74, p. 182-190, 2009.

GRAIPEL, M. E.; GOULART, F. V. B.; TORTATO, M. A.; SANTOS, L. G. R.; GHIZONI-JR., I. R. Como preservar nossos valores naturais? Ciência Hoje, Rio de Janeiro, v. 42, p. 66-69, 2008.

GREGORIN, R. Taxonomia e variação geográfica das espécies do gênero Alouatta Lacépède (Primates, Atelidae) no Brasil. Revista Brasileira de Zoologia, Curitiba, v. 23, n. 1, p. 64-144, 2006.

IBGE - INSTITUTO BRASILEIRO DE GEOGRAFIA E ESTATÍSTICA. Mapa da vegetação do Brasil. Brasília: IBGE, 2004. Disponível em: <ftp://ftp.ibge.gov.br/Cartas_e_Mapas/ Mapas_Murais/>.Acesso em: 14 maio 2011.

IGNIS - PLANEJAMENTO E IN-FORMAÇÃO AMBIENTAL. Lista das espécies da fauna ameaçadas de extinção em Santa Catarina. Itajaí: IGNIS, 2010. Disponível em $<$ http://ignis.org.br/ lista/>. Acesso em: 02 dezembro 2010.

IUCN - INTERNATIONAL UNION FOR CONSERVATION OF NATURE AND NATURAL RESOURCES. IUCN Red list of threatened species. Versão 2010.4. Disponível em: <www. iucnredlist.org>. Acesso em: 5 dez. 2010.

KIERULFF, M. C.; STALLINGS, J. R.; SÁBATO, E. L. A method to capture the bamboo rat (Kannabateomys amblyonyx) in bamboo forests. Mammalia, Paris, v. 55, p. 633-635, 1991.

KLEIN, R. M. Fisionomia, importância e recursos da vegetação do Parque Estadual da Serra do Tabuleiro. Sellowia, Itajaí, v. 33, p. 5-54, 1981.

KUHNEN, V. V.; LIMA, R. E. M.; SANTOS, J. F.; GRAIPEL, M. E.; MACHADO FILHO, L. C. P.; SORIANO-SIERRA, E. J. First record of Leopardus pardalis (Linnaeus, 1758) at the State Park of the Serra do Tabuleiro, Santa Catarina, Brazil. Brazilian Journal of Biology (online), São Carlos, v. 71, n. 1, p. 219-220, 2011.

MALCOLM, J. R. Comparative abundances of Neotropical small mammals by trap height. Journal of Mammalogy, Lawrence, v. 72, n. 1, p. 188-192, 1991.

MARTINS, M.; DIAS, D.; FRANÇA, D. S.; BOLZAN, D. P.; PEREIRA, S. N.; PERACCHI, A. L. Análise preliminar da diversidade de morcegos de Floresta Montana do Parque Nacional do Itatiaia, estado do Rio de Janeiro, Brasil. Chiroptera Neotropical, Brasília, v. 16, n. 1, Supl., p. 128-130, 2010.

MMA - MINISTÉRIO DO MEIO AMBIENTE. Lista da fauna brasileira ameaçada de extinção. Brasília: MMA, 2003. Disponível em: <http://www.mma.gov.br/port/sbf/fauna $>$. Acesso em: 4 ago. 2004.

MODESTO, T.; PESSÔA, F. S.; ENRICI, M. C.; ATTIAS, N.; JORDÃO-NOGUEIRA, T.; COSTA, L. M.; ALBUQUERQUE, H. G.; BERGALlO, H. G. Mamíferos do Parque Estadual do Desengano, Rio do Janeiro, Brazil. Biota Neotropica, Campinas, v. 8, n. 4, p. 153-159, 2008.

MONTEIRO-FILHO, E. L. A.; GRAIPEL, M. E. Captura e marcação. In: CÁCERES, N. C.; MONTEIRO-FILHO, E. L. A (Eds). Marsupiais do Brasil: Biologia, Ecologia e Evolução. Campo Grande: Editora UFMS, 2006. p. 17-27.

OLIVEIRA-SANTOS, L. G. R.; TORTATO, M. A.; GRAIPEL, M. E. Activity pattern of Atlantic Forest small arboreal mammals as revealed by camera traps. Journal of Tropical Ecology, Cambridge, v. 24, p. 563-567, 2008.
PERCEQUILLO, A. R.; WEKSLER, M.; COSTA, L. P. A new genus and species of rodent from the Brazilian Atlantic Forest (Rodentia: Cricetidae: Sigmodontinae: Oryzomyini), with comments on oryzomyine biogeography. Zoological Journal of the Linnean Society, London, v. 161, p. 357-390, 2011.

PEST - PARQUE ESTADUAL DA SERRA DO TABULEIRO. Parque Estadual da Serra do Tabuleiro, retratos da fauna e flora. Florianópolis: criAG, 2009. 79 p.

QUADROS, J.; CÁCERES, N. C. Ecologia e conservação de mamíferos na Reserva Volta Velha, SC, Brasil. Acta Biológica Leopoldensia, São Leopoldo, v. 23, n. 2, p. 213-224, 2001.

REIS, N. R.; PERACCHI, A. L.; PEDRO, W. A.; LIMA, I. P. (Eds). Mamíferos do Brasil. Londrina: Nélio R. dos Reis, 2006. 437 p.

REITZ, R.; ROSARIO, L. A.; SCHMITZ, R. J. Restauração da fauna desaparecida na Baixada do Maciambu. Sellowia, Série Zoologia, Itajaí, v. 2, p. 5-182, 1982.

ROSÁRIO, L. A. A natureza do Parque Estadual da Serra do Tabuleiro. Florianópolis: FATMA, 2003. 128 p.

SALVADOR, C. H.; FERNANDEZ, F. A. S. Population dynamics and conservation status of the insular cavy Cavia intermedia (Rodentia: Caviidae). Journal of Mammalogy, Lawrence, v. 89, n. 3, p. 721-729, 2008a.

SALVADOR, C. H.; FERNANDEZ, F. A. S. Reproduction and growth of a rare, island-endemic cavy (Cavia intermedia) from southern Brazil. Journal of Mammalogy, Lawrence, v. 89, n. 4, p. 909-915, 2008b.

SCHNEIDER, M.; MARQUES, A. A. B.; LIMA, R. S. S.; NOGUEIRA, C. P.; PRINTES, R. C.; SILVA, J. A. S. Lista atualizada dos mamíferos encontrados no Parque Nacional de Serra da Canastra (MG) e arredores, com comentários sobre as espécies. Biociências, Porto Alegre, v. 8, n. 2, p. 3-17, 2000.

SICURO, F. L.; OLIVEIRA, L. F. B. Skull morphology and functionality of extant Felidae (Mammalia: Carnivora): a phylogenetic and evolutionary perspective. Zoological Journal of the Linnean Society, London, v. 161, n. 2, p. 414-462, 2011.

STALLINGS, J. R.; FONSECA, G. A. B.; SOUZA PINTO, L. P.; SOUZA GUIAR, L. M.; SÁBATO, E. L. Mamíferos do Parque Florestal Estadual do Rio Doce, Minas Gerais, Brasil. Revista Brasileira de Zoologia, Curitiba, v. 7, p. 663-677, 1991.

TORTATO, M. A.; CAMPBELL-THOMPSON, E. R. Ocupação de caixas de nidificação por vertebrados de pequeno porte em área de Floresta Atlântica no sul do Brasil, e sua viabilidade de uso. Biotemas, Florianópolis, v. 19, n. 2, p. 67-75, 2006.

VOSS, R. S.; EMMONS, L. H. Mammalian diversity in Neotropical lowland rainforests: A preliminary assessment. Bulletin of the American Museum of Natural History, New York, v. 230, p. 1-115, 1996.

VOSS, R. S.; JANSA, S. A. Phylogenetic relationships and classification of didelphid marsupials, an extant radiation of New World metatherian mammals. Bulletin of the American Museum of Natural History, New York, v. 322, p. 1-177, 2009.

WEKSLER, M.; PERCEQUILlO, A. R.; VOSS, R. S. Ten new genera of oryzomyine rodents (Cricetidae: Sigmodontinae). American Museum Novitates, New York, v. 3537, p. 1-29, 2006. 


\section{ANEXO 1}

Exemplares procedentes dos municípios e ilhas que compõem o Parque Estadual da Serra do Tabuleiro depositados na Coleção Científica de Mamíferos da Universidade Federal de Santa Catarina (UFSC). Outros exemplares estão citados em Cherem et al. (2004).

Chironectes minimus: São Bonifácio (UFSC 3503).

Cryptonanus sp.: Palhoça (UFSC 3849, 3850, 4465).

Didelphis albiventris: São Bonifácio (UFSC 3572, 3673).

Didelphis aurita: Santo Amaro da Imperatriz (UFSC 3452).

Gracilinanus microtarsus: Santo Amaro da Imperatriz (UFSC 3214).

Marmosa paraguayana: Palhoça (UFSC 3848, 4588); Santo Amaro da Imperatriz (UFSC 3289).

Monodelphis iheringi: Santo Amaro da Imperatriz (UFSC 857, 858, 3533, 3797-3798, 4055, 45864587).

Monodelphis scalops: Santo Amaro da Imperatriz (UFSC 3344).

Dasypus novemcinctus: Palhoça (UFSC 3362); Santo Amaro da Imperatriz (UFSC 3453).

Molossus molossus: Santo Amaro da Imperatriz (UFSC 3310).

Cerdocyon thous: Palhoça (UFSC 3065); Paulo Lopes (UFSC 3101).

Leopardus tigrinus: Palhoça (UFSC 3528); Santo Amaro da Imperatriz (UFSC 3795).

Galictis cuja: Águas Mornas (UFSC 870); São Bonifácio (UFSC 3360).

Nasua nasua: São Bonifácio (UFSC 3480).

Procyon cancrivorus: Palhoça (UFSC 3358).

Tapirus terrestris: São Bonifácio (UFSC 3024).

Abrawayaomys ruschi: Santo Amaro da Imperatriz (UFSC 3427).
Akodon montensis: Palhoça (UFSC 3103, 4594); Santo Amaro da Imperatriz (3164-3168, 3200, 3209, 3218, 3219, 3270-3275, 3405-3409, 3450-3451).

Brucepattersonius iheringi: Santo Amaro da Imperatriz (UFSC 3282-3284, 3424-3425).

Delomys sublineatus: Santo Amaro da Imperatriz (UFSC 3170, 3287-3288, 4213).

Drymoreomys albimaculatus: (UFSC 860).

Euryoryzomys russatus: Santo Amaro da Imperatriz (UFSC 3210, 3212-3213, 3285-3286, 3348-3349, 3356, 3419-3422).

Holochilus sp.: Palhoça (UFSC 4099, 4425, 4426, 4593).

Nectomys squamipes: Palhoça (UFSC 4104).

Oligoryzomys flavescens: Palhoça (UFSC 4497-4499, 4504, 4507, 4509, 4514-4515, 4519-4520).

Oligoryzomys nigripes: Palhoça (4495, 4508, 4510, 4517-4518); Santo Amaro da Imperatriz (UFSC 3217, 3350, 3354-3355, 3414-3417).

Oxymycterus judex: Santo Amaro da Imperatriz (UFSC 3169, 3171, 3172, 3942).

Sooretamys angouya: Palhoça (4490-4491); Santo Amaro da Imperatriz (UFSC 3812).

Thaptomys nigrita: Santo Amaro da Imperatriz (UFSC 3276-3281, 3351-3552).

Cricetidae (gen. e sp. indet.): Santo Amaro da Imperatriz (UFSC 980).

Sphiggurus villosus: Palhoça (UFSC 3506).

Cavia intermedia: Ilha de Moleques do Sul (UFSC 3192-3199, 3316-3333, 3345-3347, 3363-3383, $3393-3398,3468-3479$, 3484-3490, 3496, 35393540, 3557-3565).

Cavia magna: Palhoça (UFSC 2814).

Hydrochoerus hydrochaeris: Palhoça (UFSC 3541).

Dasyprocta azarae: Paulo Lopes (UFSC 3262).

Phyllomys sulinus: Santo Amaro da Imperatriz (UFSC 2778, 4214).

Myocastor coypus: Palhoça (UFSC 3261). 